\title{
Identification of potential crucial genes and molecular mechanisms in glioblastoma multiforme by bioinformatics analysis
}

\author{
XIAOJIE CHEN $^{1}$, YUANBO PAN ${ }^{2}$, MENGXIA YAN $^{1}$, GUANSHUI BAO $^{1}$ and XUHONG SUN ${ }^{1}$ \\ ${ }^{1}$ Department of Neurology, Shanghai Ninth People's Hospital, School of Medicine, Shanghai Jiao Tong University, \\ Shanghai 201999; ${ }^{2}$ Department of Neurosurgery, Second Affiliated Hospital, School of Medicine, \\ Zhejiang University, Hangzhou, Zhejiang 310000, P.R. China
}

Received August 22, 2019; Accepted April 4, 2020

DOI: $10.3892 / \mathrm{mmr} .2020 .11160$

\begin{abstract}
Glioblastoma multiforme (GBM) is the most common and malignant brain tumor of the adult central nervous system and is associated with poor prognosis. The present study aimed to identify the hub genes in GBM in order to improve the current understanding of the underlying mechanism of GBM. The RNA-seq data were downloaded from The Cancer Genome Atlas database. The edgeR package in $\mathrm{R}$ software was used to identify differentially expressed genes (DEGs) between two groups: Glioblastoma samples and normal brain samples. Gene Ontology (GO) functional enrichment analysis and the Kyoto Encyclopedia of Genes and Genomes pathway enrichment analysis were performed using Database for Annotation, Visualization and Integrated Discovery software. Additionally, Cytoscape and Search Tool for the Retrieval of Interacting Genes/Proteins tools were used for the protein-protein interaction network, while the highly connected modules were extracted from this network using the Minimal Common Oncology Data Elements plugin. Next, the prognostic significance of the candidate hub genes was analyzed using UALCAN. In addition, the identified hub genes were verified by reverse transcription-quantitative (RT-q) PCR. In total, 1,483 DEGs were identified between GBM and control samples, including 954 upregulated genes and 529 downregulated genes $(\mathrm{P}<0.01$; fold-change $>16)$ and these genes were involved in different GO terms and signaling pathways. Furthermore, $C D K 1, B U B 1, B U B 1 B, C E N P A$ and $G N G 3$ were identified as key genes in the GBM samples. The UALCAN tool verified that higher expression level of CENPA
\end{abstract}

Correspondence to: Dr Guanshui Bao or Dr Xuhong Sun, Department of Neurology, Shanghai Ninth People's Hospital, School of Medicine, Shanghai Jiao Tong University, 280 Mohe Road, Shanghai 201999, P.R. China

E-mail: baogs@163.com

E-mail: charmesun@aliyun.com

Key words: glioblastoma multiforme, differentially expressed genes, functional enrichment analyses, Kyoto Encyclopedia of Genes and Genomes pathway, prognosis was relevant to poorer overall survival rates. In conclusion, $C D K 1, B U B 1, B U B 1 B, C E N P A$ and $G N G 3$ were found to be potential biomarkers for GBM. Additionally, 'cell cycle' and ' $\gamma$-aminobutyric acid signaling' pathways may serve a significant role in the pathogenesis of GBM.

\section{Introduction}

According to the guidelines from the World Health Organization, gliomas are categorized into grades I to IV. Glioblastoma multiforme (GBM) is classified as grade IV and it is a highly malignant form $(1,2)$. GBM has been recognized as the most aggressive type of brain tumor with highly infiltrative ability (3), affecting about 20,000 people every year in the United States (4). Currently, the treatments for GBM include surgical resection, chemotherapy and radiotherapy (5). However, due to the aggressiveness of gliomas and their resistance to chemotherapy and radiation therapy $(6,7)$, the prognosis of GBM is still poor with a median survival time of 12-15 months for patients with GBM $(8,9)$. In recent years, targeted therapies, such as combinatorial chemotherapy targeting molecular subgroups and gene and immune therapies, have made important progress in preclinical models (10-12). However, the potential molecular mechanism in the pathogenesis of GBM needs to be further investigated. In order to improve the prognosis of GBM patients, it is important to identify molecular mechanism underlying GBM pathogenesis.

In recent years, a number of studies have investigated the potential molecular mechanisms of GBM. It has been reported that the upregulation and mutations in EGFR may be responsible for the resistance of glioma cells to the treatments with chemotherapy and radiation (13-15). Additionally, Annexin-A5 over-activation increases Snail expression level via the PI3K/Akt/NF- $\kappa$ B signaling pathway, which is associated with glioblastoma cells migration and invasion (16). In addition, Siebzehnrubl et al (17) indicated that $Z E B 1$ is linked to tumor chemoresistance and invasion of glioblastoma cells by modulating its downstream effectors, including $c-M Y B, M G M T$ and $R O B O 1$. Nevertheless, the exact molecular mechanism and gene networks underlying GBM progression remain to be fully elucidated.

The present study aimed to identify the potential key nodes and molecular mechanisms associated with the progression of GBM. Co-expression interactions between the identified 
DEGs were conducted using a protein-protein interaction (PPI) network and several critical genes were identified. In addition, a network module analysis was performed according to the PPI network. In conclusion, the present study may improve the current understanding of the pathogenesis underlying GBM and identified key genes that may represent novel targets for the development of novel treatments of GBM.

\section{Materials and methods}

Data download. RNA-seq data were downloaded from The Cancer Genome Atlas (TCGA) database (portal.gdc.cancer. gov/) (18). The data of 169 GBM samples and 5 normal brain tissue samples were used and analyzed.

Data preprocessing and DEG analysis. Based on the annotation information contained in the Ensembl database (asia. ensembl.org/html) (19), 23,269 protein coding genes were identified. Then, the DEGs between GBM samples and normal samples were screened using edgeR package in $\mathrm{R}(20,21)$. A $\mathrm{P}<0.01$ and $\mid \log 2$ (fc) $\mid>4$ were selected as the cutoff criteria for the identification of DEGs. In addition, a volcano plot was drawn using the gplots package in R software (22).

Functional and pathway enrichment analysis of DEGs. To reveal the main functional pathways of GBM, the Database for Annotation, Visualization and Integrated Discovery (DAVID; https://david.ncifcrf.gov/) was used to analyze Gene Ontology (GO) terms and Kyoto Encyclopedia of Genes and Genomes (KEGG) pathways enriched in the identified DEGs (23). DEG count $\geq 2$ and $\mathrm{P}<0.05$ were used as cutoff to identify the significant biological functions and signaling pathways.

PPI network and module analysis of DEGs. The Search Tool for the Retrieval of Interacting Genes/Proteins (STRING) database (https://string-db.org/) was used to assess the interacting partners of the DEGs (24), and the PPI network was constructed with a combined score $>0.7$. Visualization of the network was performed with Cytoscape (version 3.6.1) software (25). After constructing the PPI network, topology characteristics of the network were analyzed using the Network Analyzer plug-in of Cytoscape software, and degree distribution, distribution of the shortest path, average clustering coefficient and closeness centrality were examined (26). Subsequently, the top 10 significant hub genes or key nodes were identified according to their connectivity degrees by using the CytoHubba tool, a plug-in for Cytoscape (27). The MCODE plug-in was used to analyze the highly interconnected clusters of this network with default parameters (28).

UALCAN survival analysis. UALCAN (ualcan.path.uab.edu/) is an interactive web-software that can be used to perform analyses of tumor subgroup gene expression and survival (29). In UALCAN, samples were categorized into two groups: High expression and Low/Medium expression patients. High expression patients refer to the highest quarter of all patients. Low and medium expression patients refer to the remaining three quarters of patients. The P-values from the Kaplan-Meier analysis were based on log-rank. The effects of candidate key gene expression levels on overall survival (OS) were analyzed using UALCAN.
Sample collection and ethics statement. To determine the expression of hub genes in GBM tissues, samples from 20 patients (age, 26-77 years), including nine males and eleven females, who underwent tumor resection surgery were collected at the Shanghai Ninth People's Hospital between September 2010 and March 2018. All patients provided written informed consent, and the study conducted in accordance with the Declaration of Helsinki. In addition, 20 normal brain tissues (used as the control group) were collected from patients who suffered from traumatic brain injury and required internal decompression surgery. After collection, all tissues were frozen in liquid nitrogen and stored at $-80^{\circ} \mathrm{C}$. All participants in the present study signed informed consents and the study was approved by the Ethics Review Committee of Shanghai Ninth People's Hospital.

RNA extraction and reverse transcription-quantitative (RT-q) PCR. TRIzol (cat. no. 9109; Takara Bio, Inc.) was used to isolate total RNA from each tissue sample according to the manufacturer's instructions. Briefly, $2 \mathrm{ml}$ TRIzol was added to brain tissue $(100 \mathrm{mg})$ to prepare homogenate. After obtaining RNA, TE buffer was used to dilute the RNA. Nanodrop software (ND2000C; Gene Company, Ltd.) was used to determine the OD value. A value of $\mathrm{OD}_{260} / \mathrm{OD}_{280}$ within 1.7-2.1 indicated that the extracted RNA was relatively pure. Then, total RNA was reverse transcribed into cDNAs using the PrimeScript RT Master Mix (cat. no. RR036A; Takara Bio, Inc.). RT-qPCR was performed to measure the levels of cDNAs using a SYBR GREEN kit (cat. no. RR420 A; Takara Bio, Inc.). qPCR amplification procedure was performed as follows: Pre-denaturation at $95^{\circ} \mathrm{C}$ for $30 \mathrm{sec}$ for 1 cycle followed by 40 cycles of $95^{\circ} \mathrm{C}$ for $5 \mathrm{sec}$ and $60^{\circ} \mathrm{C}$ for $20 \mathrm{sec}$. The relative expression level of the five key genes was calculated following comparative CT method, as previously described (30). GAPDH was used to normalize the mRNA expression level. The primer sequences are presented in Table I.

Statistical analysis. Experimental data are presented as the mean \pm standard error of the mean. All statistical analyses were performed using SPSS 20.0 (IBM, Corp.) and visualized using GraphPad Prism 6.0 (GraphPad Software Inc.). Differences in expression levels between groups were analyzed by unpaired Student's t-test. $\mathrm{P}<0.05$ was considered to indicate a statistically significant difference.

\section{Results}

Identification of DEGs. Using $\mathrm{P}<0.01$ as a cutoff, a total of 1,483 DEGs (954 upregulated genes and 529 downregulated) were identified between GBM and control samples (Table S1). The volcano plot of DEGs is presented in Fig. 1. Each individual dot represents a DEG.

Functional and pathway enrichment analysis of the DEGs. The GO enrichment analysis results revealed that 954 upregulated DEGs were primarily involved in biological process that included 'anterior/posterior pattern specification', 'embryonic skeletal system morphogenesis', 'sister chromatid cohesion' and 'cell division' (Fig. 2). According to the 'cellular components' analysis, 
Table I. Primer sequences for PCR.

\begin{tabular}{lll}
\hline cDNA & \multicolumn{1}{c}{ Forward primer $\left(5^{\prime}-3 '\right)$} & Reverse primer $\left(5^{\prime}-3^{\prime}\right)$ \\
\hline$C D K 1$ & CACAAAACTACAGGTCAAGTGG & GAGAAATTTCCCGAATTGCAGT \\
$B U B 1$ & GAAAGCATGAGCAATGGGTAAA & CCACCTGATGCAACTTCTTATG \\
$B U B 1 B$ & ATGGGTCCTTCTGGAAACTTAG & GGAATGTAGTGTCAAAAACCCC \\
$C E N P A$ & AAGAGCACACACCTCTTGATAA & CATGTAAGGTGAGGAGATAGGC \\
$G A P B$ & CGGTGAACAGCACTATGAGTAT & TCACAGTAAGTCATCAGGTCTG \\
& GTGACCTGACCTGCCGTCTAG & GAGTGGGTGTCGCTGTTGAAGTC
\end{tabular}

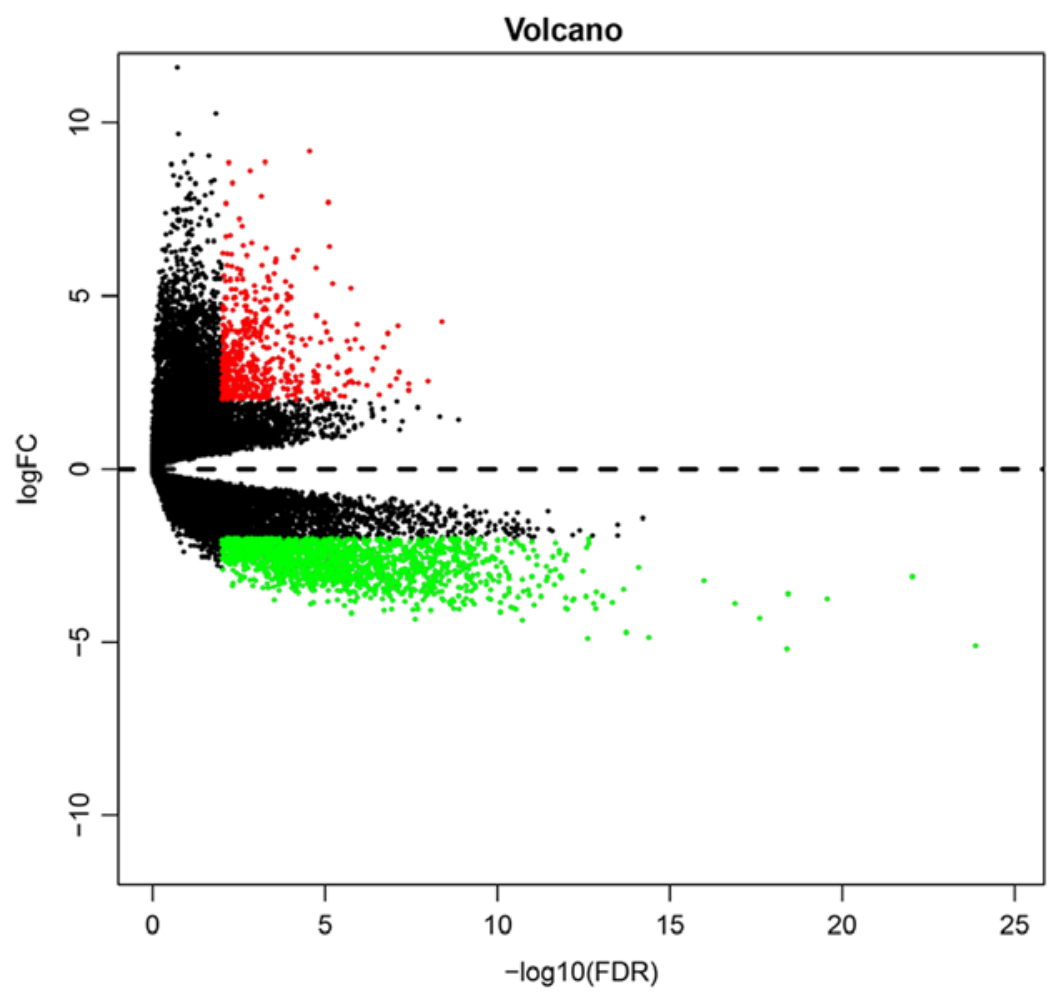

Figure 1. Volcano plot of differentially expressed genes in glioblastoma multiform. Black, non-differentially expressed genes; red, significantly upregulated genes; green, significantly downregulated genes (based on $\mid \log 2 \mathrm{FCl}>4$ and $\mathrm{P}<0.01$ ). FC, fold-change. FDR, false discovery rate.

DEGs were found to be primarily localized in the nucleus. In addition, the most significantly enriched molecular functions were related to transcriptional activator activity. In addition, as presented in Fig. 3, 529 downregulated DEGs were significantly associated with 'chemical synaptic transmission', 'regulation of ion transmembrane transport' and ' $\gamma$-aminobutyric acid signaling pathway'. The top five main biological processes enriched in the upregulated and downregulated DEGs are presented in Tables II and III, respectively. The associated signaling pathways significantly enriched in the identified DEGs are presented in Table IV. The KEGG pathway analysis indicated that upregulated DEGs were mostly involved in pathways such as 'cell cycle' and 'p53 signaling' pathways, whereas downregulated genes were associated with 'retrograde endocannabinoid signaling', 'GABAergic synapse' and 'glutamatergic synapse'.

PPI network construction of DEGs. Based on the STRING database and Cytoscape software, PPI networks were constructed with combined scores $>0.7$, including 645 nodes and 1,779 edges (Fig. 4). Subsequently, the node degree distribution of this network was analyzed using a pattern of power-law according to the topology property (Fig. 5). In this network, a degree value $>40$ was used as the cutoff criterion for the CytoHubba tool. Finally, 10 DEGs were identified as the hub genes with the highest connectivity degree: cyclin dependent kinase $1(C D K 1)$, centromere protein A $(C E N P A)$, $\mathrm{G}$ protein subunit $\gamma 3(G N G 3)$, BUB1 mitotic checkpoint serine/threonine kinase $(B U B 1)$, cyclin $\mathrm{B} 2(C C N B 2)$, kinesin family member $2 \mathrm{C}(K I F 2 C)$, aurora kinase B $(A U R K B)$, baculoviral IAP repeat containing 5 (BIRC5), cell division cycle associated $8(C D C A 8)$ and BUB1 mitotic checkpoint serine/threonine kinase $\mathrm{B}(B U B 1 B)$.

Module analysis of the PPI network. Generally, biological networks contain several functional modules and they may interact in various biological process (31). In the present study, 
A

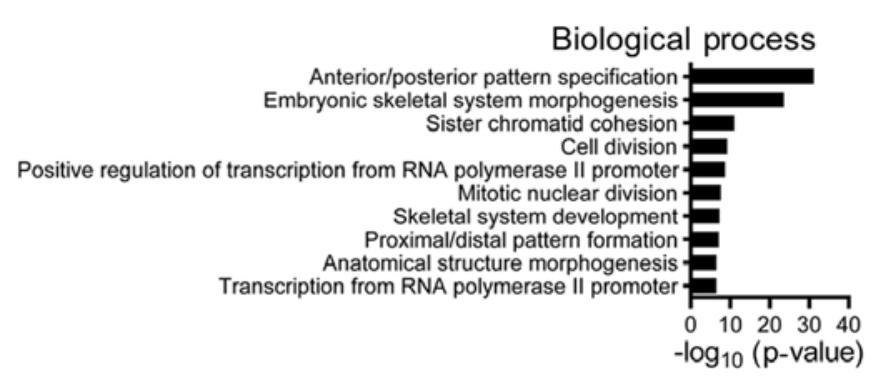

C

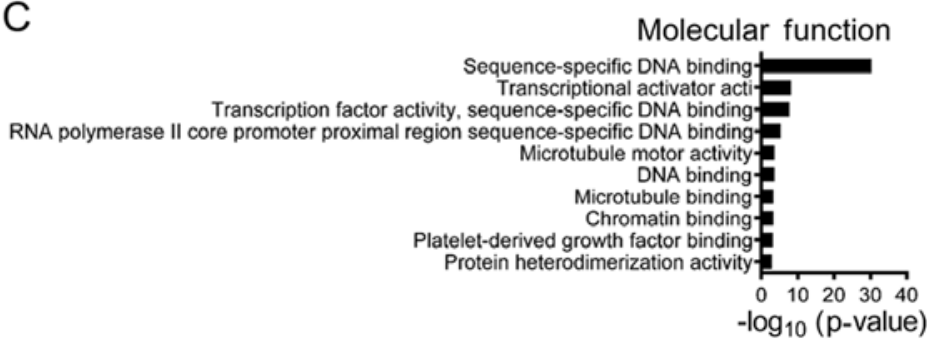

B

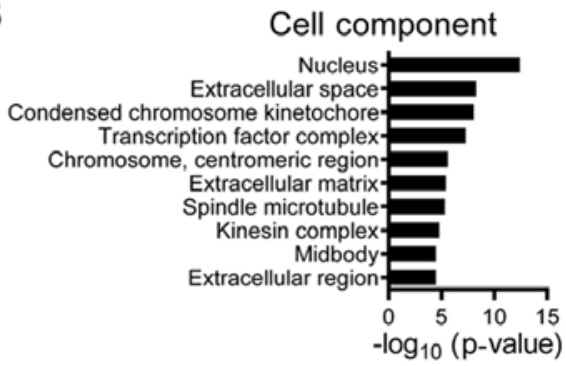

Figure 2. Top 10 Gene Ontology analysis of 954 upregulated differentially expressed genes associated with glioblastoma multiforme. (A) 'Biological Processes', (B) 'Cellular Components' and (C) 'Molecular Function', and (D) KEGG pathway analysis. KEGG, Kyoto Encyclopedia of Genes and Genomes.

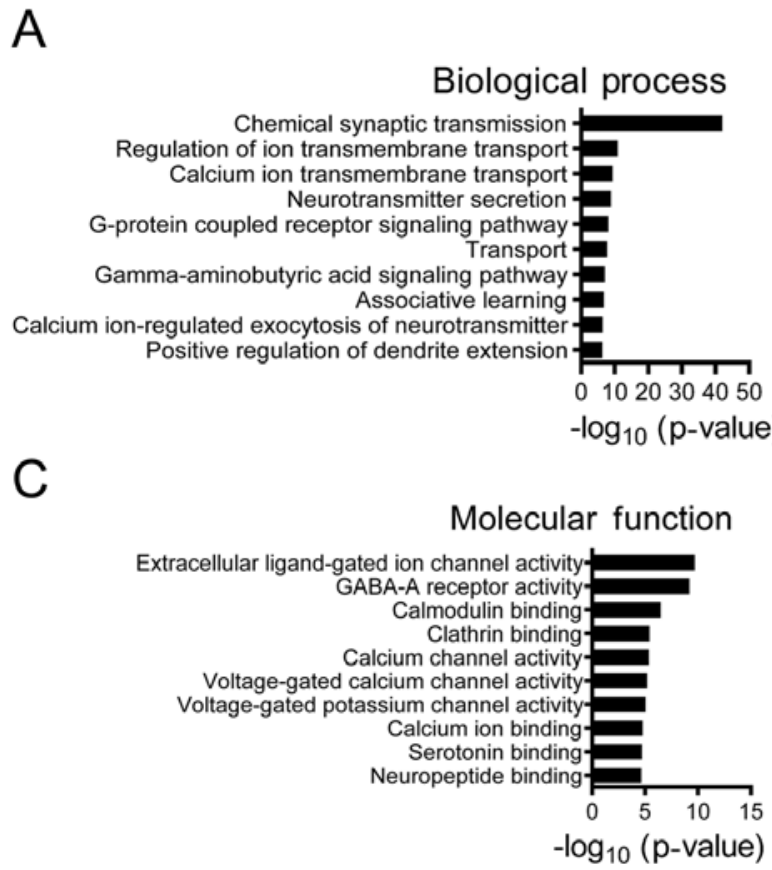

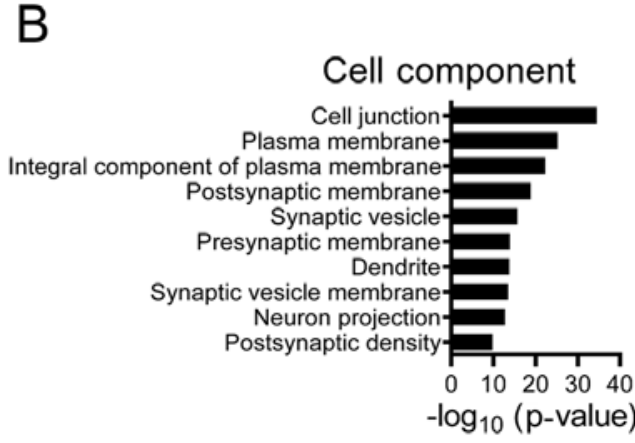

$\mathrm{D}$

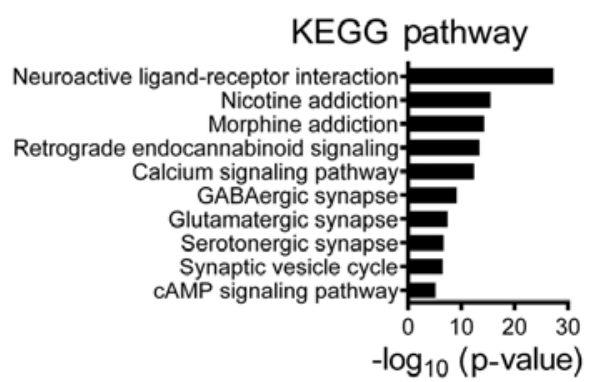

Figure 3. Top 10 Gene Ontology analysis of 529 downregulated differentially expressed genes associated with glioblastoma multiforme. (A) 'Biological Processes', (B) 'Cellular Components' and (C) 'Molecular Function', and (D) KEGG pathway analysis. KEGG, Kyoto Encyclopedia of Genes and Genomes.

the top two clusters were identified from the PPI network using MCODE analysis. The most significant cluster involved 35 nodes and 572 edges, and $C D K 1, B U B 1, B U B 1 B, C E N P A$ were highly enriched (Fig. 6A). Cluster 2 is presented in Fig. 6B, consisting of 24 nodes and 276 edges. Specifically, the hub gene GNG3 was enriched in cluster 2, which indicated that it may be involved in the pathogenesis of GBM by cooperating with other genes.

UALCAN survival analysis. UALCAN was used to analyze the OS of 169 patients with GBM based on TCGA data.
Briefly, survival analysis indicated that the higher expression of CENPA gene was significantly correlated with shorter OS time in GBM patients, whereas the expression of $C D K 1, B U B 1$, $B U B 1 B$ and $G N G 3$ was not correlated with OS (Fig. 7).

Expression of hub genes in GBM. To further verify the expression level of hub genes in GBM samples, RT-qPCR was performed to calculate the mRNA levels of the five hub genes identified in the present study $(C D K 1, B U B 1, B U B 1 B$, $C E N P A$ and $G N G 3$ ) in GBM samples. As illustrated in Fig. 8, the expression of $C D K 1, B U B 1, B U B 1 B$ and $C E N P A$ were 
Table II. GO analysis of upregulated genes associated with glioblastoma multiforme.

\begin{tabular}{|c|c|c|c|}
\hline Category & Term & Count & P-value \\
\hline GOTERM_BP_DIRECT & GO:0007062 sister chromatid cohesion & 17 & $8.70 \mathrm{E}-12$ \\
\hline GOTERM_BP_DIRECT & GO:0051301 cell division & 26 & $5.28 \mathrm{E}-10$ \\
\hline GOTERM_BP_DIRECT & GO:0007067 mitotic nuclear division & 20 & $2.20 \mathrm{E}-08$ \\
\hline GOTERM_BP_DIRECT & GO:0007051 spindle organization & 3 & 0.026829 \\
\hline GOTERM_BP_DIRECT & GO:0007094 mitoticspindle assembly checkpoint & 3 & 0.04073 \\
\hline GOTERM_CC_DIRECT & GO:0005634 nucleus & 142 & $3.58 \mathrm{E}-13$ \\
\hline GOTERM_CC_DIRECT & GO:0005615 extracellular space & 51 & $5.11 \mathrm{E}-09$ \\
\hline GOTERM_CC_DIRECT & GO:0000777 condensed chromosome kinetochore & 13 & $9.06 \mathrm{E}-09$ \\
\hline GOTERM_MF_DIRECT & GO:0043565 sequence-specific DNA binding & 57 & $5.42 \mathrm{E}-31$ \\
\hline GOTERM_MF_DIRECT & GO:0003677 DNA binding & 46 & $2.76 \mathrm{E}-04$ \\
\hline GOTERM_MF_DIRECT & GO:0003682 chromatin binding & 17 & $2.76 \mathrm{E}-04$ \\
\hline
\end{tabular}

GO, Gene Ontology; BP, 'Biological Process'; CC, 'Cellular Component'; MF, 'Molecular Function'.

Table III. GO analysis of downregulated genes associated with glioblastoma multiforme.

\begin{tabular}{llr}
\hline Category & \multicolumn{1}{c}{ Term } & Count \\
\hline GOTERM_BP_DIRECT & GO:0007268 chemical synaptic transmission & 54 \\
GOTERM_BP_DIRECT & GO:0034765 regulation of ion transmembrane transport & $1.09 E-42$ \\
GOTERM_BP_DIRECT & GO:0007269 neurotransmitter secretion & 1.37 -11 \\
GOTERM_BP_DIRECT & GO:0007187 G-protein coupled receptor signaling pathway, coupled & 18 \\
& to cyclic nucleotide second messenger & $1.33 E-09$ \\
GOTERM_BP_DIRECT & GO:0007214 gamma-aminobutyric acid signaling pathway & $7.02 E-09$ \\
GOTERM_CC_DIRECT & GO:0005886 plasma membrane & 8 \\
GOTERM_CC_DIRECT & GO:0005887 integral component of plasma membrane & $7.68 E-08$ \\
GOTERM_CC_DIRECT & GO:0045211 postsynaptic membrane & $7.00 E-26$ \\
GOTERM_MF_DIRECT & GO:0005230 extracellular ligand-gated ion channel activity & $4.55 E-23$ \\
GOTERM_MF_DIRECT & GO:0004890 GABA-A receptor activity & 85 \\
GOTERM_MF_DIRECT & GO:0005516 calmodulin binding & 32 \\
\hline
\end{tabular}

GO, Gene Ontology; BP, biological process; CC, cellular component; MF, molecular function.

significantly upregulated in GBM samples compared with normal control tissues. By contrast, the expression level of GNG3 was higher in the control tissues compared with tumor tissues. The present RT-qPCR results were in line with the aforementioned bioinformatics analysis, suggesting that these key genes may be linked to the molecular mechanism underlying GBM.

\section{Discussion}

Glioblastoma multiforme (GBM) is the most common aggressive brain tumor, and is associated with a poor patient survival rate (32). However, the most important challenge in treating GBM is the presence of significant intra-tumor heterogeneity $(13,33,34)$. Although previous studies have reported numerous potential biomarkers associated with the progression of GBM, the potential molecular mechanism underlying its pathogenesis has not been comprehensively investigated (35-37). In the present study, a total of 1,483 DEGs were identified, containing 954 upregulated genes and 529 downregulated genes. The present results suggested that these DEGs were primarily enriched in cell division, mitotic nuclear division and chemical synaptic transmission. In addition, the upregulated and downregulated genes were related to cell cycle, p53 signaling pathway and synapse. A PPI network was constructed and the highly connected module were identified. After module analysis, several hub genes with higher degree of connectivity were identified, including $C D K 1, B U B 1, B U B 1 B$, CENPA and GNG3.

In the TCGA database, there were only 5 control samples. Although it is of a smaller size when compared with the 169 GBM samples, it should not have great influence on the results of the present study, as there was relatively little individual difference in the control simples from different patients, whereas the difference was much more evident in tumor samples due to intratumoral heterogeneity between different patients. 
Table IV. Results of KEGG pathway analysis.

A, Upregulated genes

\begin{tabular}{lcc}
\hline Term & Count & P-value \\
\hline hsa04110: Cell cycle & 12 & $3.26 \mathrm{E}-07$ \\
hsa05322: Systemic lupus erythematosus & 9 & $2.49 \mathrm{E}-04$ \\
hsa04115: p53 signaling pathway & 6 & 0.001428 \\
hsa04512: ECM-receptor interaction & 6 & 0.004496 \\
hsa05202: Transcriptional misregulation in cancer & 8 & 0.004710 \\
\hline
\end{tabular}

B, Downregulated genes

\begin{tabular}{lcc}
\hline Term & Count & P-value \\
\hline hsa04080: Neuroactive ligand-receptor interaction & 44 & $5.35 \mathrm{E}-28$ \\
hsa04723: Retrograde endocannabinoid signaling & 20 & $4.27 \mathrm{E}-14$ \\
hsa04020: Calcium signaling pathway & 24 & $3.72 \mathrm{E}-13$ \\
hsa04727: GABAergic synapse & 15 & $7.40 \mathrm{E}-10$ \\
hsa04724: Glutamatergic synapse & 15 & $3.76 \mathrm{E}-08$ \\
\hline
\end{tabular}

KEGG, Kyoto Encyclopedia of Genes and Genomes.

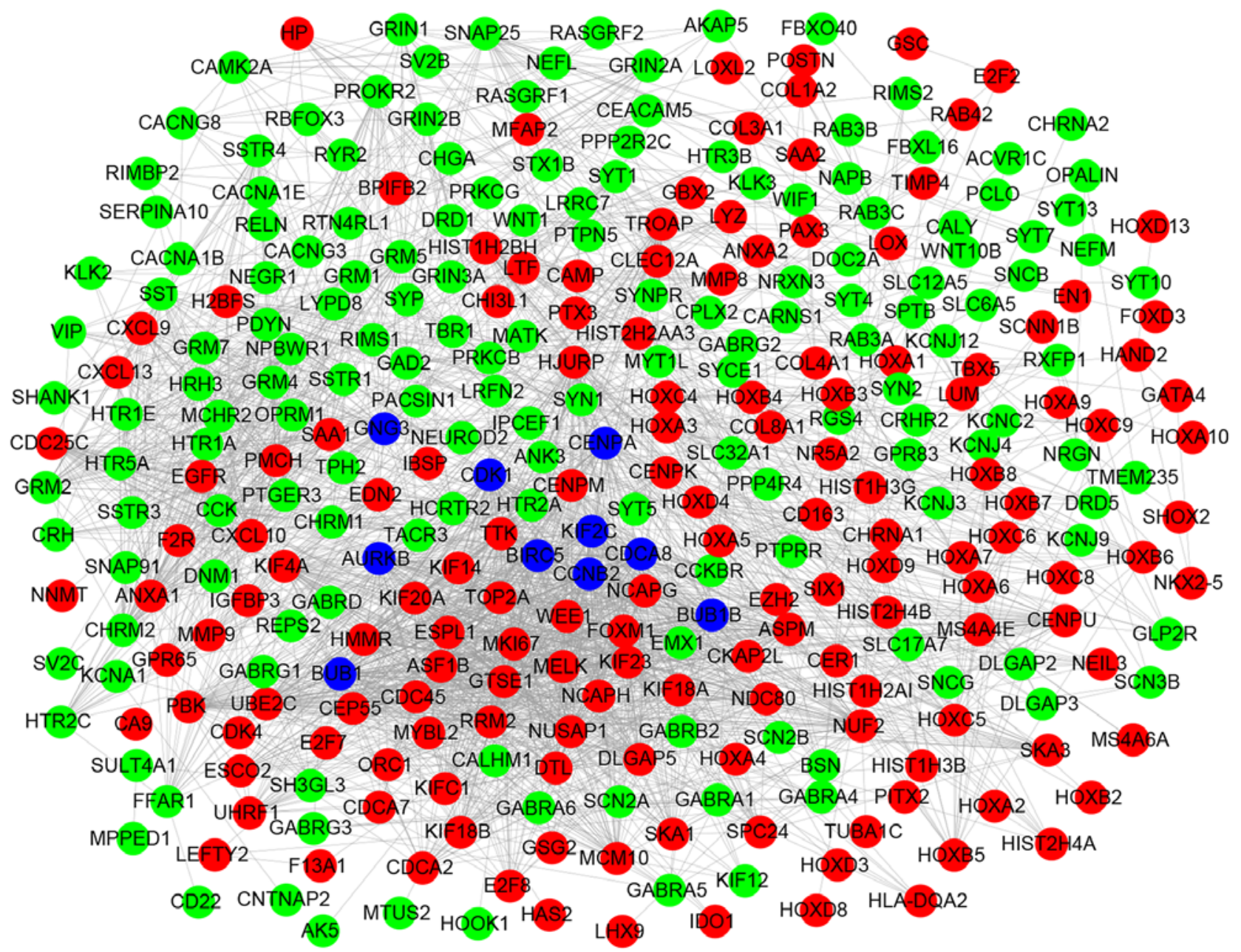

Figure 4. The giant protein-protein interaction network. Key nodes in the network are presented in different colors: Blue stands for the hub genes, red corresponds to the upregulated genes and green corresponds to the downregulated genes in glioblastoma multiforme. 

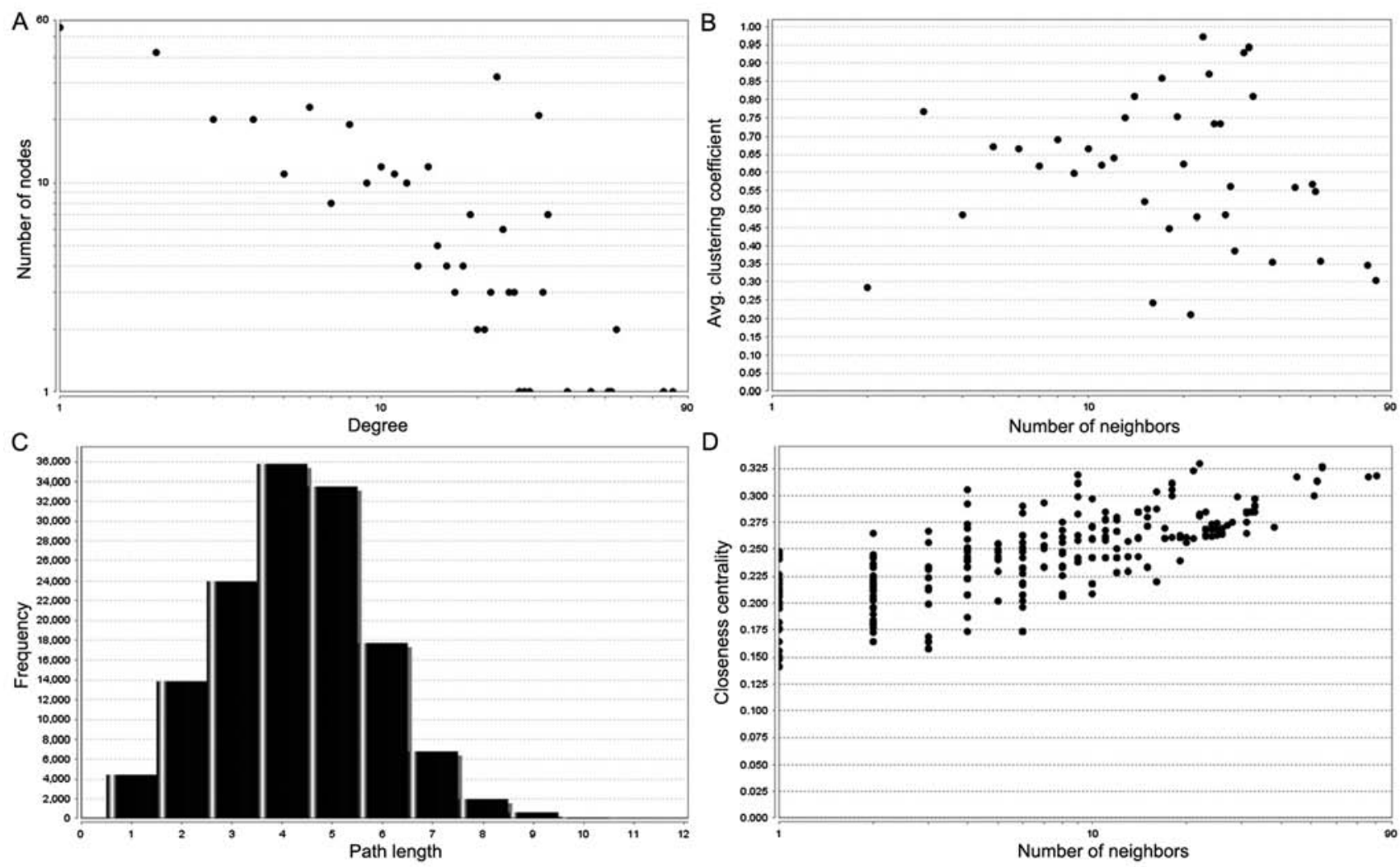

Figure 5. The topology properties. (A) Distribution of degrees, (B) Average clustering coefficient, (C) Shortest path distribution and (D) Closeness centrality.
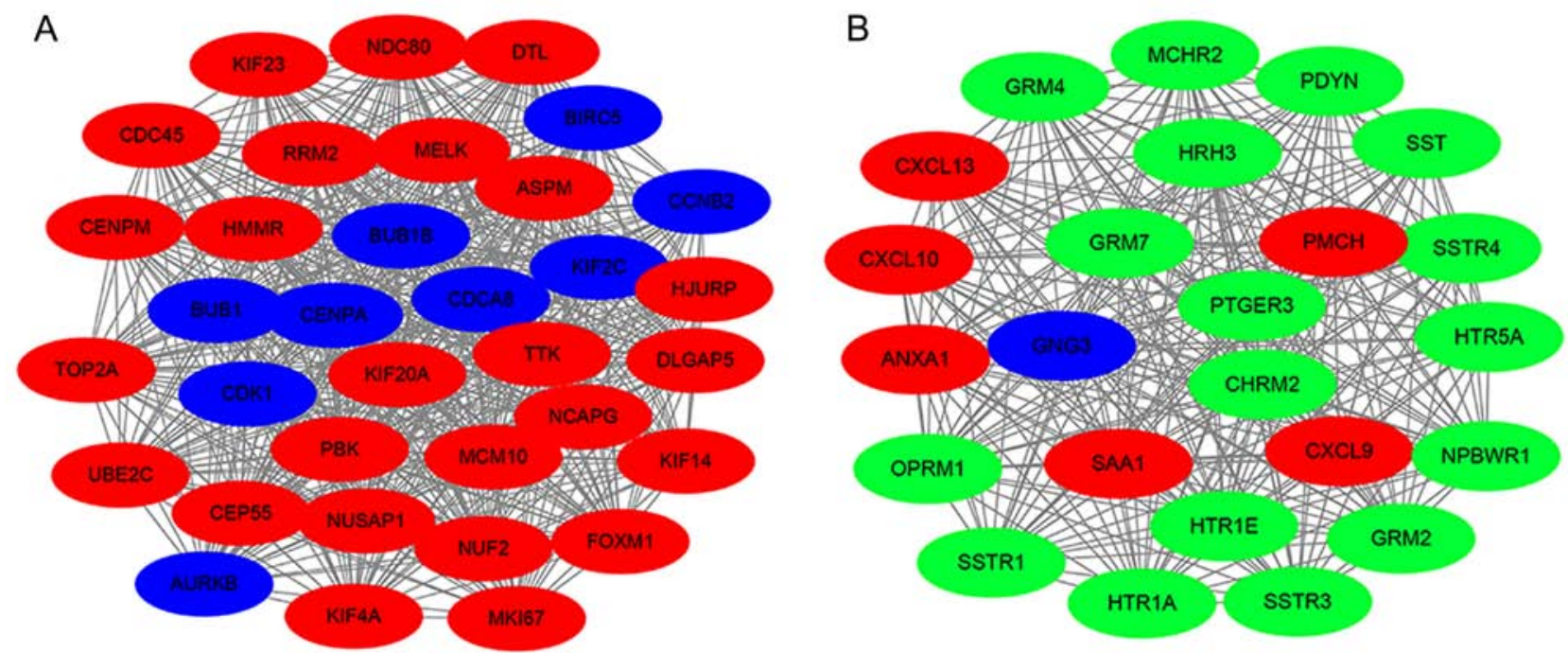

Figure 6. Top 2 modules were extracted from the protein-protein interaction network. Blue stands for the hub genes, red corresponds to the upregulated genes and green corresponds to the downregulated genes in glioblastoma multiforme. (A) Cluster 1 and (B) cluster 2.

$C D K 1$, also named cell division control protein 2 , is an important cell cycle regulator functioning as a serine/threonine kinase $(38,39)$. During $\mathrm{G} 2$ and early mitosis, active CDK1-cyclin complexes can phosphorylate various downstream proteins, leading to re-organization of the cytoskeleton, nuclear envelope breakdown and chromosome condensation $(40,41)$. Previous studies have reported that downregulation of $C D K 1$ could inhibit proliferative ability of human glioma cells, while overexpression of $C D K 1$ contributed to senescence escape of the cells and promoted oncogenesis of human gliomas (42-44). In the present study, $C D K 1$ was also observed to be significantly upregulated in the module analysis of the PPI network constructed. The present results suggested that $C D K 1$, interacting with other genes identified in the present module analysis, may be associated with the progression of GBM by modulating the cell cycle.

In addition, $C D K 1, B U B 1, B U B 1 B$ and $C E N P A$ were also identified as key nodes in the current study. $B U B 1$ and $B U B 1 B$ are spindle assembly checkpoint (SAC) genes that serve as a controller of mitotic checkpoints and chromosome segregation $(32,45)$. BUB1 mRNA was reported to be upregulated 

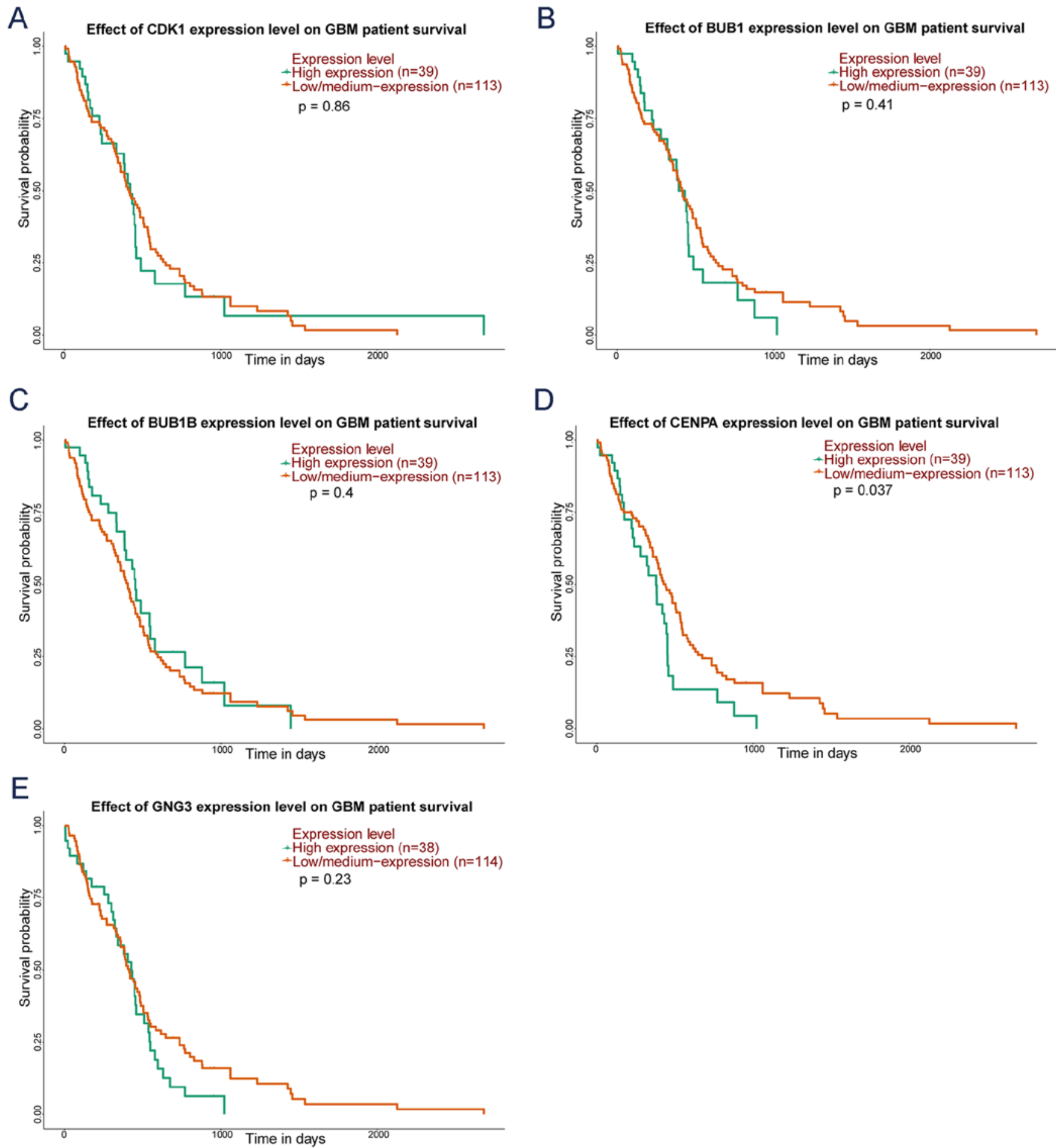

Figure 7. Kaplan-Meier curve of five key genes in patients with GBM. The red lines represent patients with low gene expression, and green lines represent patients with high gene expression. (A) $C D K 1$, (B) BUB1, (C) BUB1B, (D) CENPA and (E) GNG3. GBM, glioblastoma multiforme. CDK1, cyclin dependent kinase 1; $B U B 1$, BUB1 mitotic checkpoint serine/threonine kinase; $B U B 1 B$, BUB1 mitotic checkpoint serine/threonine kinase B; $C E N P A$, centromere protein A; GNG3, G protein subunit $\gamma 3$.

in glioma samples and the expression level was positively correlated with glioma grade (46). In addition, $B U B 1 B$ was identified to be enriched in glioblastoma cells and associated with radio-resistance and recurrence of glioblastoma $(47,48)$. In accordance with these previous studies, the present study identified that the expression levels of both $B U B 1$ and $B U B 1 B$ in GBM samples were higher compared with the negative controls. Collectively, accumulating evidence suggested that $B U B I$ and
$B U B 1 B$ may exert a significant influence on the progression of GBM by regulating mitotic spindle assembly checkpoint and sister chromosome segregation during mitosis. It has been previously reported that SAC inhibition promoted the response of glioblastoma cells to antimitotic drugs and enhanced the efficacy of tumor treating fields, which impair mitosis by disturbing the spindle formation $(49,50)$. Therefore, inhibition of $B U B 1$ or $B U B 1 B$ may be a therapeutic strategy to treat GBM by 

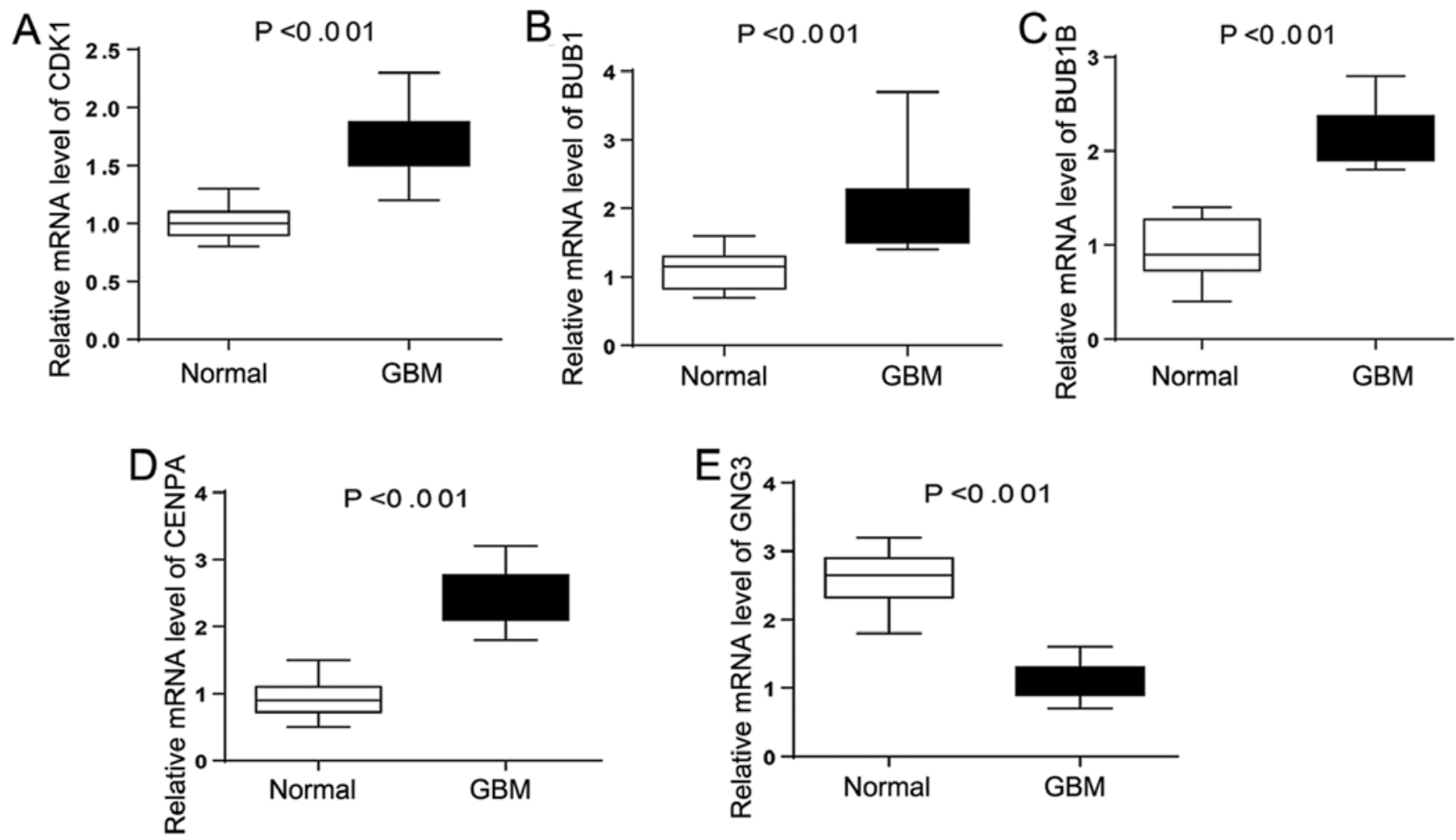

Figure 8. The mRNA expression of five hub genes in tissues of GBM patients. (A) CDK1, (B) BUB1, (C) BUB1B, (D) CENPA and (E) GNG3. Expression of these genes was normalized against GAPDH expression. The statistical significance of differences was analyzed by t-test. GBM, glioblastoma multiforme. $C D K 1$, cyclin dependent kinase $1 ; B U B 1$, BUB1 mitotic checkpoint serine/threonine kinase; $B U B 1 B$, BUB1 mitotic checkpoint serine/threonine kinase B; CENPA, centromere protein A; GNG3, G protein subunit $\gamma 3$.

influencing SAC during the process of cell division. In addition, CENPA, which encodes a centromere-associated protein, plays an important role in cell division by directing the assembly of active kinetochores (51). Failure in this process could lead to dysfunction of chromosome segregation, which has been found to be associated with initiation and progression of cancer (52-54). Notably, the present study identified a higher expression level of CENPA in brain tissue samples from GBM patients and high levels of CENPA were significantly associated with shorter OS. In addition, $\mathrm{GO}$ enrichment analyses showed that CENPA was involved in the biological function of sister chromatid cohesion, nucleosome assembly and mitotic cytokinesis. The present results suggested that $C E N P A$ gene may regulate the mitosis of glioma cells by interacting with other genes in the cluster 1 , thus suggesting an association with pathogenesis and prognosis in GBM. The present study identified a novel potential target molecule in GBM, and inhibition of CENPA may be a novel potential therapeutic strategy for GBM.

The present results showed that $G N G 3$ may be associated with the GABAergic synapse pathway. GNG3 is a gene that encodes the $\gamma$ subunits of $\mathrm{G}$ proteins (55), and the disruption of GNG3 may induce the dysfunction of the GABAB1 receptor signaling pathway (56). Notably, a previous study reported that switching $G A B A$ catabolism toward $\gamma$-hydroxybutyric acid production could suppress glioblastoma cell tumorigenic properties (57). Therefore, it was hypothesized that low expression of GNG3 may be associated with the pathogenesis of GBM by regulating related signaling pathways (Table SII). However, further studies are required to confirm the role of GNG3 in GBM.
Collectively, the present study has identified that several hub genes (CDK1, BUB1, BUB1B, CENPA and GNG3) were involved in the mechanism of GBM. The present results suggested that $C D K 1, B U B 1, B U B 1 B$ and $C E N P A$ may play an essential role in the cell cycle pathway, leading to GBM progression. In addition, GNG3 was found to be a potential therapeutic target to treat GBM. The present results may provide novel insights into the molecular mechanisms of GBM. Additional studies investigating the hub genes identified in the present study are required to examine their detailed function and interaction in GBM. However, several classical molecular traits, such as IDH1 mutation status, were not analyzed with these hub genes in our study. The authors believe that the IDH mutation analysis for these hub genes, such as CENPA, need to be investigated in the future. Furthermore, it is also appealing to investigate whether these hub genes are regulated by oncogenic mutations of themselves or other genes in the future.

\section{Acknowledgements}

Not applicable.

\section{Funding}

This research was financially supported by The Shanghai Science \& Technology Research Program (grant no. 14411972200) and Shanghai Wu Mengchao Medical Science Foundation (grant no. JJHM-2019009). 


\section{Availability of data and materials}

The datasets analyzed in the present study are available from The Cancer Genome Atlas database (https://portal.gdc.cancer.gov/).

\section{Authors' contributions}

GB and XS conceived and designed the study. YP downloaded and analyzed the RNA-seq data. XC and MY collected the samples. XC and MY analyzed the data. XC wrote the manuscript. All authors read and approved the manuscript and agree to be accountable for all aspects of the research in ensuring that the accuracy or integrity of any part of the work are appropriately investigated and resolved.

\section{Ethics approval and consent to participate}

Informed consent was obtained from all patients and the present study was approved by the Ethics Review Committee of Shanghai Ninth People's Hospital.

\section{Patient consent for publication}

Not applicable.

\section{Competing interests}

The authors declare that they have no competing interest.

\section{References}

1. Louis DN, Ohgaki H, Wiestler OD, Cavenee WK, Burger PC, Jouvet A, Scheithauer BW and Kleihues P: The 2007 WHO classification of tumours of the central nervous system. Acta Neuropathol 114: 97-109, 2007

2. Louis DN, Perry A, Reifenberger G, von Deimling A, Figarella-Branger D, Cavenee WK, Ohgaki H, Wiestler OD, Kleihues P and Ellison DW: The 2016 World Health Organization classification of tumors of the central nervous system: A summary. Acta Neuropathol 131: 803-820, 2016.

3. Kloosterhof NK, de Rooi JJ, Kros M, Eilers PH, Sillevis Smitt PA, van den Bent MJ and French PJ: Molecular subtypes of glioma identified by genome-wide methylation profiling. Gene Chromosomes Cancer 52: 665-674, 2013.

4. Rao SS, Lannutti JJ, Viapiano MS, Sarkar A and Winter JO: Toward 3D biomimetic models to understand the behavior of glioblastoma multiforme cells. Tissue Eng Part B Rev 20: 314-327, 2014.

5. Alifieris C and Trafalis DT: Glioblastoma multiforme: Pathogenesis and treatment. Pharmacol Ther 152: 63-82, 2015.

6. Yang B, Ma YB and Chu SH: Silencing SATB1 overcomes temozolomide resistance by downregulating MGMT expression and upregulating SLC22A18 expression in human glioblastoma cells. Cancer Gene Ther 25: 309-316, 2018.

7. Doan NB, Nguyen HS, Al-Gizawiy MM, Mueller WM, Sabbadini RA, Rand SD, Connelly JM, Chitambar CR, Schmainda KM and Mirza SP: Acid ceramidase confers radioresistance to glioblastoma cells. Oncol Rep 38: 1932-1940, 2017.

8. Martinez R, Rohde V and Schackert G: Different molecular patterns in glioblastoma multiforme subtypes upon recurrence. J Neurooncol 96: 321-329, 2010.

9. Witthayanuwat S, Pesee M, Supaadirek C, Supakalin N Thamronganantasakul K and Krusun S: Survival analysis of glioblastoma multiforme. Asian Pac J Cancer Prev 19: 2613-2617, 2018

10. Herrlinger U, Tzaridis T, Mack F, Steinbach JP, Schlegel U, Sabel M, Hau P, Kortmann RD, Krex D, Grauer O, et al: Lomustine-temozolomide combination therapy versus standard temozolomide therapy in patients with newly diagnosed glioblastoma with methylated MGMT promoter (CeTeG/NOA-09): A randomised, open-label, phase 3 trial. Lancet 393: 678-688, 2019.
11. Hashemi M,Hadjighassem M,Zali A and Hashemi J: Tumoricidal effect of human olfactory ensheathing cell mediated suicide gene therapy in human glioblastoma cells. Mol Biol Rep 45: 2263-2273, 2018.

12. Chao CN, Yang YH, Wu MS, Chou MC, Fang CY, Lin MC, Tai CK, Shen CH, Chen PL, Chang D and Wang M: Gene therapy for human glioblastoma using neurotropic JC virus-like particles as a gene delivery vector. Sci Rep 8: 2213, 2018.

13. Nathanson DA, Gini B, Mottahedeh J, Visnyei K, Koga T, Gomez G, Eskin A, Hwang K, Wang J, Masui K, et al: Targeted therapy resistance mediated by dynamic regulation of extrachromosomal mutant EGFR DNA. Science 343: 72-76, 2014.

14. Zhou K, Yao H, Zhang X, Liu J, Qi Z, Xie X, Xu X, Zhou Y, Yu Z, Wang Z, et al: Next generation sequencing and molecular imaging identify EGFR mutation and amplification in a glioblastoma multiforme patient treated with an EGFR inhibitor: A case report. Oncotarget 8: 50305-50313, 2017.

15. Hatanpaa KJ, Burma S, Zhao D and Habib AA: Epidermal growth factor receptor in glioma: Signal transduction, neuropathology, imaging, and radioresistance. Neoplasia 12: 675-684, 2010.

16. Ji C, Guo H, Zhang P, Kuang W, Fan Y and Wu L: AnnexinA5 promote glioma cell invasion and migration via the PI3K/Akt/NF-кB signaling pathway. J Neurooncol 138: 469-478, 2018.

17. Siebzehnrubl FA, Silver DJ, Tugertimur B, Deleyrolle LP, Siebzehnrubl D, Sarkisian MR, Devers KG, Yachnis AT, Kupper MD, Neal D, et al: The ZEB1 pathway links glioblastoma initiation, invasion and chemoresistance. EMBO Mol Med 5: 1196-1212, 2013.

18. Liu J, Liu D, Yang Z and Yang Z: High LAMC1 expression in glioma is associated with poor prognosis. OncoTargets Ther 12: 4253-4260, 2019

19. Yates AD, Achuthan P, Akanni W, Allen J, Allen J, Alvarez-Jarreta J, Amode MR, Armean IM, Azov AG, Bennett R, et al: Ensembl 2020. Nucleic Acids Res 48: D682-D688, 2020.

20. Robinson MD, McCarthy DJ and Smyth GK: edgeR: A Bioconductor package for differential expression analysis of digital gene expression data. Bioinformatics 26: 139-140, 2010.

21. Anders S, McCarthy DJ, Chen Y, Okoniewski M, Smyth GK, Huber W and Robinson MD: Count-based differential expression analysis of RNA sequencing data using R and Bioconductor. Nat Protoc 8: 1765-1786, 2013.

22. Warnes GR, Bolker B, Bonebakker L, Gentleman R, Huber W, Liaw A, Lumley T, Maechler M, Magnusson A, Moeller S, et al: gplots: Various R programming tools for plotting data. R Package Version 2.4, 2009.

23. Dennis G Jr, Sherman BT, Hosack DA, Yang J, Gao W, Lane HC and Lempicki RA: DAVID: Database for Annotation, Visualization, and Integrated Discovery. Genome Biol 4: P3, 2003.

24. Szklarczyk D, Franceschini A, Wyder S, Forslund K, Heller D, Huerta-Cepas J, Simonovic M, Roth A, Santos A, Tsafou KP, et al: STRING v10: Protein-protein interaction networks, integrated over the tree of life. Nucleic Acids Res 43: D447-D452, 2015.

25. Diaz-Montana JJ, Gomez-Vela F and Diaz-Diaz N: GNC-app: A new Cytoscape app to rate gene networks biological coherence using gene-gene indirect relationships. Biosystems 166: 61-65, 2018.

26. Shannon P, Markiel A, Ozier O, Baliga NS, Wang JT, Ramage D, Amin N, Schwikowski B and Ideker T: Cytoscape: A software environment for integrated models of biomolecular interaction networks. Genome Res 13: 2498-2504, 2003.

27. Chin CH, Chen SH, Wu HH, Ho CW, Ko MT and Lin CY: cytoHubba: Identifying hub objects and sub-networks from complex interactome. BMC Syst Biol 8 (Suppl 4): S11, 2018.

28. Bandettini WP, Kellman P, Mancini C, Booker OJ, Vasu S, Leung SW, Wilson JR, Shanbhag SM, Chen MY and Arai AE: MultiContrast delayed enhancement (MCODE) improves detection of subendocardial myocardial infarction by late gadolinium enhancement cardiovascular magnetic resonance: A clinical validation study. J Cardiovasc Magn Reson 14: 83, 2012.

29. Chandrashekar DS, Bashel B, Balasubramanya SAH, Creighton CJ, Ponce-Rodriguez I, Chakravarthi BVSK and Varambally S: UALCAN: A Portal for Facilitating Tumor Subgroup Gene Expression and Survival Analyses. Neoplasia 19: 649-658, 2017.

30. Livak KJ and Schmittgen TD: Analysis of relative gene expression data using real-time quantitative PCR and the 2(-Delta Delta C(T)) method. Methods 25: 402-408, 2001. 
31. Li L, Chen X and Chen Z: Identification of key candidate genes in dairy cow in response to escherichia coli mastitis by bioinformatical analysis. Front Genet 10: 1251, 2019.

32. Watkins $\mathrm{S}$ and Sontheimer H: Unique biology of gliomas: Challenges and opportunities. Trends Neurosci 35: 546-556, 2012

33. Teng J, da Hora CC, Kantar RS, Nakano I, Wakimoto H, Batchelor TT, Chiocca EA, Badr CE and Tannous BA: Dissecting inherent intratumor heterogeneity in patient-derived glioblastoma culture models. Neuro Oncol 19: 820-832, 2017.

34. Bonavia R, Inda MM, Cavenee WK and Furnari FB: Heterogeneity maintenance in glioblastoma: A social network. Cancer Res 71: 4055-4060, 2011.

35. Xiao S, Yang Z, Lv R, Zhao J, Wu M, Liao Y and Liu Q: miR-135b contributes to the radioresistance by targeting GSK3 $\beta$ in human glioblastoma multiforme cells. PLoS One 9: e108810, 2014.

36. Zhu J, Ye J, Zhang L, Xia L, Hu H, Jiang H, Wan Z, Sheng F, Ma Y, Li W, et al: Differential expression of circular RNAs in glioblastoma multiforme and its correlation with prognosis. Transl Oncol 10: 271-279, 2017.

37. Song YC, Lu GX, Zhang HW, Zhong XM, Cong XL, Xue SB, Kong R, Li D, Chang ZY, Wang XF, et al: Proteogenomic characterization and integrative analysis of glioblastoma multiforme. Oncotarget 8: 97304-97312, 2017.

38. Fourest-Lieuvin A, Peris L, Gache V, Garcia-Saez I, Juillan-Binard C, Lantez V and Job D: Microtubule regulation in mitosis: Tubulin phosphorylation by the cyclin-dependent kinase Cdk1. Mol Biol Cell 17: 1041-1050, 2006.

39. Malumbres $M$ and Barbacid $M$ : Cell cycle, CDKs and cancer: A changing paradigm. Nat Rev Cancer 9: 153-166, 2009.

40. Malumbres $M$ and Barbacid M: Mammalian cyclin-dependent kinases. Trends Biochem Sci 30: 630-641, 2005.

41. Nigg EA: Mitotic kinases as regulators of cell division and its checkpoints. Nat Rev Mol Cell Biol 2: 21-32, 2001.

42. Li W, Liu J, Fu W, Zheng X, Ren L, Liu S, Wang J, Ji T and Du G: 3 -O-acetyl-11-keto- $\beta$-boswellic acid exerts anti-tumor effects in glioblastoma by arresting cell cycle at G2/M phase. J Exp Clin Cancer Res 37: 132, 2018.

43. Song Z, Pan Y, Ling G, Wang S, Huang M, Jiang X and Ke Y: Escape of U251 glioma cells from temozolomide-induced senescence was modulated by CDK1/survivin signaling. Am J Transl Res 9: 2163-2180, 2017.

44. Chen H, Huang Q, Dong J, Zhai DZ, Wang AD and Lan Q: Overexpression of CDC2/CyclinB1 in gliomas, and CDC2 depletion inhibits proliferation of human glioma cells in vitro and in vivo. BMC Cancer 8: 29, 2008.

45. de Voer RM, Geurts van Kessel A, Weren RD, Ligtenberg MJ, Smeets D, Fu L, Vreede L, Kamping EJ, Verwiel ET, Hahn MM et al: Germline mutations in the spindle assembly checkpoint genes BUB1 and BUB3 are risk factors for colorectal cancer. Gastroenterology 145: 544-547, 2013.

46. Bie L, Zhao G, Cheng P, Rondeau G, Porwollik S, Ju Y, Xia XQ and McClelland M: The accuracy of survival time prediction for patients with glioma is improved by measuring mitotic spindle checkpoint gene expression. PLoS One 6: e25631, 2011.
47. Ma Q, Liu Y, Shang L, Yu J and Qu Q: The FOXM1/BUB1B signaling pathway is essential for the tumorigenicity and radioresistance of glioblastoma. Oncol Rep 38: 3367-3375, 2017.

48. Lee E, Pain M, Wang H, Herman JA, Toledo CM, DeLuca JG, Yong RL, Paddison P and Zhu J: Sensitivity to $B U B 1 B$ inhibition defines an alternative classification of glioblastoma. Cancer Res 77: 5518-5529, 2017.

49. Tannous BA, Kerami M, Van der Stoop PM, Kwiatkowski N, Wang J, Zhou W, Kessler AF, Lewandrowski G, Hiddingh L, Sol N, et al: Effects of the selective MPS1 inhibitor MPS1-IN-3 on glioblastoma sensitivity to antimitotic drugs. J Natl Cancer Inst 105: 1322-1331, 2013.

50. Kessler AF, Frombling GE, Gross F, Hahn M, Dzokou W, Ernestus RI, Löhr M and Hagemann C: Effects of tumor treating fields (TTFields) on glioblastoma cells are augmented by mitotic checkpoint inhibition. Cell Death Discov 4: 12, 2018.

51. De Rop V, Padeganeh A and Maddox PS: CENP-A: The key player behind centromere identity, propagation, and kinetochore assembly. Chromosoma 121: 527-538, 2012.

52. Tomonaga T, Matsushita K, Yamaguchi S, Oohashi T, Shimada H, Ochiai T, Yoda K and Nomura F: Overexpression and mistargeting of centromere protein-A in human primary colorectal cancer. Cancer Res 63: 3511-3516, 2003.

53. Rajput $\mathrm{AB}, \mathrm{Hu} \mathrm{N}$, Varma S, Chen $\mathrm{CH}$, Ding K, Park PC, Chapman JA, Sengupta SK, Madarnas Y, Elliott BE and Feilotter HE: Immunohistochemical assessment of expression of centromere protein-A (CENPA) in Human invasive breast cancer. Cancers 3: 4212-4227, 2011.

54. Li YM, Liu XH, Cao XZ, Wang L and Zhu MH: Expression of centromere protein A in hepatocellular carcinoma. Zhonghua Bing Li Xue Za Zhi 36: 175-178, 2007 (In Chinese).

55. Downes GB, Copeland NG, Jenkins NA and Gautam N: Structure and mapping of the $\mathrm{G}$ protein gamma3 subunit gene and a divergently transcribed novel gene, gng3lg. Genomics 53: 220-230, 1998

56. Schwindinger WF, Giger KE, Betz KS, Stauffer AM, Sunderlin EM, Sim-Selley LJ, Selley DE, Bronson SK and Robishaw JD: Mice with deficiency of $\mathrm{G}$ protein gamma3 are lean and have seizures. Mol Cell Biol 24: 7758-7768, 2004.

57. El-Habr EA, Dubois LG, Burel-Vandenbos F, Bogeas A Lipecka J, Turchi L, Lejeune FX, Coehlo PL, Yamaki T, Wittmann BM, et al: A driver role for GABA metabolism in controlling stem and proliferative cell state through GHB production in glioma. Acta Neuropathol 133: 645-660, 2017.

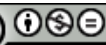

This work is licensed under a Creative Commons Attribution-NonCommercial-NoDerivatives 4.0 International (CC BY-NC-ND 4.0) License. 